\title{
THE PREVALENCE OF REPAIR IN STUDIES OF LANGUAGE EVOLUTION
}

\author{
ASHLEY MICKLOS ${ }^{* 1,2}$, VINICIUS MACUCH SILVA ${ }^{3}$, and NICOLAS FAY ${ }^{4}$ \\ *Corresponding Author: ashleymmicklos@gmail.com \\ ${ }^{1}$ Linguistics Department, University of California, San Diego, USA \\ ${ }^{2}$ Language and Cognition Department, Max Planck Institute for \\ Psycholinguistics, Nijmegen, The Netherlands \\ ${ }^{3}$ Department of Linguistics, University of Tübingen, Germany \\ ${ }^{4}$ School of Psychology, University of Western Australia, Perth, Australia
}

While studies of language evolution have themselves evolved to include interaction as a feature of interest (Healey et al, 2007; Tamariz et al, 2017; Fay et al, 2017; Byun et al, in press), many still fail to consider just what interaction offers emerging communication systems. That is, while it's been acknowledged that face-to-face interaction in communication games is beneficial in its approximation of natural language use (Macuch Silva \& Roberts, 2016; Nölle et al, 2017), there remains a lack of detailed analysis of what this type of interaction affords participants, and how those affordances impact the evolving language. To this end, here we will expose one particular process that occurs in interaction: repair, or the processes by which we can indicate misunderstanding and resolve problems in communication (Schegloff, Jefferson, \& Sacks, 1977; Jefferson, 1972). Though it is often not explicitly analyzed, repair is a relevant aspect of interaction to consider for its effects on the evolution of a communication system as well as how it demonstrates the moment-to-moment processing and negotiation of alignment in emerging communication.

We present data from various studies of language evolution in which we document how repair is carried out, the types of repair present, and their effect on novel signaling. All studies in this collection utilized referential communication tasks - some iterated over simulated generations and other repeating interactions between two individuals. However, they differ in modality (of stimuli and communication). The data collection includes: silent gesture communication of written nouns and verbs; non-linguistic vocalizations and 
gestures communication of either images or sounds; graphical communication of written nouns; and, text-based communication of tangram stimuli. Crucially, the stimuli are meant to be easily confusable. Repairs were categorized according to Dingemanse and Enfield's (2015) schema on other-initiated repair (OIR), or when a Matcher (Receiver) initiates a repair sequence that the Director (Sender) is meant to resolve.

In experiments with face-to-face interaction, repair sequences account for at least $20 \%$ of all turns. Even when participants are not face-to-face yet the task affords contingent, bidirectional communication, repair sequences take up almost $10 \%$ of all turns. These instances of repair are not trivial. We provide a descriptive analysis of how repairs are performed, and the outcomes of these repair sequences, which promote efficient, informative signals. The prevalence of these interactional sequences across studies of language evolution, and indeed across modalities, demonstrates that users of a novel communication mode make use of repair for the purposes of overcoming miscommunication and to establish alignment (with one another and to signal-meaning matches that eventually conventionalize).

Moreover, it has been argued that feedback can boost communicative efficiency, but not communication success (Fay et al, 2017). Results from a meta-analysis show that repair does indeed improve communicative efficiency when compared with non-repair trials or conditions, resulting in shorter, less complex gestural signals, more abstract and less complex drawings, and more concise textual descriptions. However, the effect of repair on communicative success has been less clear. We suggest that the affordances of certain repair types are more likely to improve communication success over other types (e.g. repairs that improve on prior signals, rather than lead to new innovations), and that repair sequences have more downstream effects on accuracy as opposed to in-situ success.

A goal of this review is to draw attention to the prevalence of repair in experimental investigations of language evolution. Repair, as a pragmatic universal (Evans and Levinson, 2009; Dingemanse et al, 2015) underlying human communication, is a mechanism that promotes efficient and successful communication. We find repair is ubiquitous across modalities and - even when not being directly tested - it is a factor that arises in, and affects the processes of, emergent communication. More broadly, we hope to call attention to not only the need to consider interaction as an ecologically valid site for language evolution and use, but also to consider the specific mechanisms within interaction that drive language to be structured as it is. 


\section{Acknowledgements}

AM thanks her co-authors, and members of the LEvInSoN group at the Max Planck Institute for Psycholinguistics for their feedback. VMS thanks his Master's thesis committee: Seán G. Roberts, Judith Holler, and Asli Özyürek.

\section{References}

Byun, K.S., de Vos, C., Bradford, A., Zeshan, U., \& Levinson, S.C. (In Press). First encounters: Repair sequences in cross-signing. Topics in Cognitive Science.

Dingemanse, M., \& Enfield, N.J. (2015). Other-initiated repair across languages: Towards a typology of conversational structures. Open Linguistics 1 (1). 96-118.

Dingemanse, M., Roberts, S. G., Baranova, J., Blythe, J., Drew, P., Floyd, S., ... Enfield, N. J. (2015). Universal principles in the repair of communication problems. PLoS ONE, 10(9), 1-15.

Evans, N., \& Levinson, S. C. (2009). The myth of language universals: language diversity and its importance for cognitive science. The Behavioral and Brain Sciences, 32(5), 429-448-494.

Fay, N., Walker, B., \& Swoboda, N. (2017). Deconstructing social interaction: The complimentary roles of behavior alignment and partner feedback to the creation of shared symbols. Proceedings of the 39th Annual Meeting of the Cognitive Science Society, July 26-29, London, United Kingdom.

Healey, P.G.T., Swoboda, N., Umata, I., \& King, J. (2007). Graphical language games: interactional constraints on representational form. Cognitive Science, 31 (2), 285-309.

Jefferson, G. (1972). Side sequences. In D. Sudnow (Ed.), Studies in Social Interaction (pp. 294-338). The Free Press: New York.

Macuch Silva V. \& Roberts S. (2016) Exploring the Role of Interaction in the Emergence of Linguistic Structure. In S. Roberts \& G. Mills (Eds.) Proceedings of EvoLang XI, Language Adapts to Interaction Workshop, 21 March, 2016.

Nölle, J., Staib, M., Fusaroli, R., \& Tylén, K. (2017). Informational bottlenecks lead to co-evolution of categories and systematicity in an emergent communication system. Proceedings of Protolang 5, Barcelona, September 2017.

Schegloff, E.A., Jefferson, G., \& Sacks, H. (1977). The preference for selfcorrection in the organization of repair in conversation. Language, 53 (2), 361-382.

Tamariz, M., Roberts, S. G., Mart, J. I., \& Santiago, J. (2017). The Interactive Origin of Iconicity. Cognitive Science, 1-16. 